\title{
Analysis of Pirlimycin Residues in Beef Muscle, Milk and Honey by a Biotin-Streptavidin-Amplified Enzyme-Linked Immunosorbent Assay
}

Wenxiao Jiang, ${ }^{\dagger}$ Ross C. Beier, ${ }^{\ddagger}$ Pengjie Luo,${ }^{\S}$ Peng Zhai, ${ }^{\dagger}$ Nan Wu,${ }^{\S}$ Guimiao Lin,${ }^{\dagger}$ Xiaomei Wang, ${ }^{*}, \dagger$ and Gaixia $\mathrm{Xu}^{\#}$

$\dagger$ School of Medicine, Health Science Center, Shenzhen University, Shenzhen, 518060, China

$\ddagger$ Food and Feed Safety Research Unit, Southern Plains Agricultural Research Center, Agricultural Research Service, USDA, 2881 F\&B Road, College Station, Texas 77845, USA

\# Key Laboratory of Food Safety Risk Assessment, Ministry of Health, China National Center for Food Safety Risk Assessment, Beijing, 100021, China

$\S$ Institute of Optoelectronics, Department of Optoelectronic Information Science and Engineering, Shenzhen University, Shenzhen, 518060, China

*Corresponding Author: Tel: +86-0755-8667-1908; Fax: +86-0755-8667-1906;

E-mail: xmwang@szu.edu.cn 


\section{LC-MS/MS analysis}

An UltiMate 3000 XRS Rapid Separation Liquid Chromatography coupled to TSQ Quantiva Mass Spectrometer (Thermo Scientific, NY) was used to analyze the samples.

LC Profile. The chromatographic separation was performed using a Hypersil GOLD column $(1.9 \mu \mathrm{m}, 50 \mathrm{~mm} \times 2.1 \mathrm{~mm}$ id., Thermo Scientific, NY). The mobile phase constituted of solvent A $(0.1 \%$ formic acid in water $)$ and solvent $\mathrm{B}$ (acetonitrile). The gradient profile was as follows: started with 10\% B (hold time 0.5 min); continued with linear change to $40 \% \mathrm{~B}$ up to $1.5 \mathrm{~min}$, and to $90 \% \mathrm{~B}$ up to 2.5 min; continued $90 \%$ B up to $3.5 \mathrm{~min}$; returned to initial condition at $3.51 \mathrm{~min}$, followed by equilibration until $6 \mathrm{~min}$. The injection volume was $15 \mu \mathrm{L}$. The flow rate was $0.3 \mathrm{~mL} / \mathrm{min}$ and the temperature was maintained at $30^{\circ} \mathrm{C}$.

MS Conditions. Detection was performed with electrospray ionization operating in positive ion mode and the tandem spectrometer was operated in the select reactions monitoring (SRM) mode. The following optimized parameters were applied in the mass spectrometer: capillary voltage, $3.5 \mathrm{kV}$; ion transport tube temperature, $350{ }^{\circ} \mathrm{C}$; vaporizer temperature, $400{ }^{\circ} \mathrm{C}$; sheath gas pressure, $40 \mathrm{psi}$; auxiliary gas pressure, 10 psi; ion sweep gas pressure, 5 psi; collision gas Pressure, 1.5 mTorr; RF Lens, $65 \mathrm{~V}$; cycle time, $0.5 \mathrm{sec}$. The precursor and products ions for each residue, along with the corresponding collision energies, are listed in Table S2. 


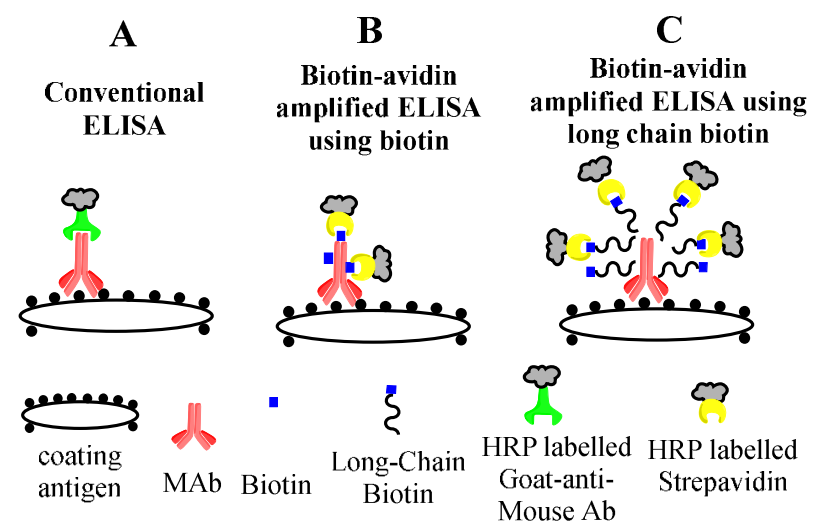

Figure S1. Schematic description of a conventional ELISA (A), the biotin-avidinamplified ELISA (B), and the long-chain biotin based biotin-avidin-amplified ELISA (C). 
A

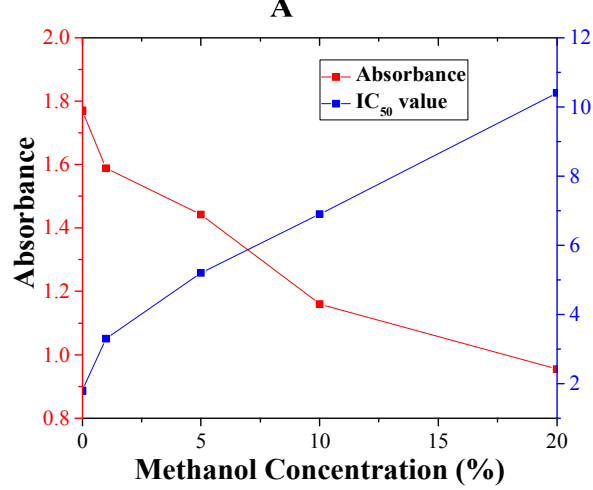

Methanol Concentration (\%)

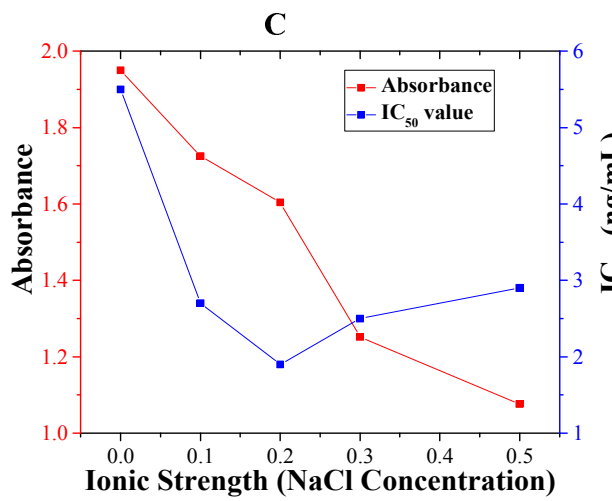

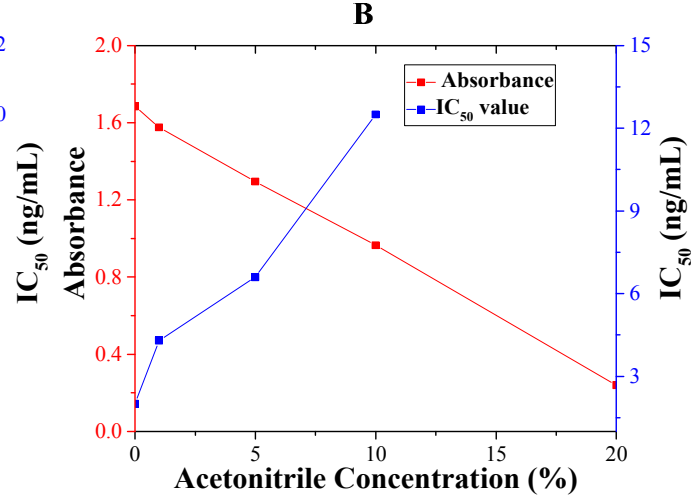

D

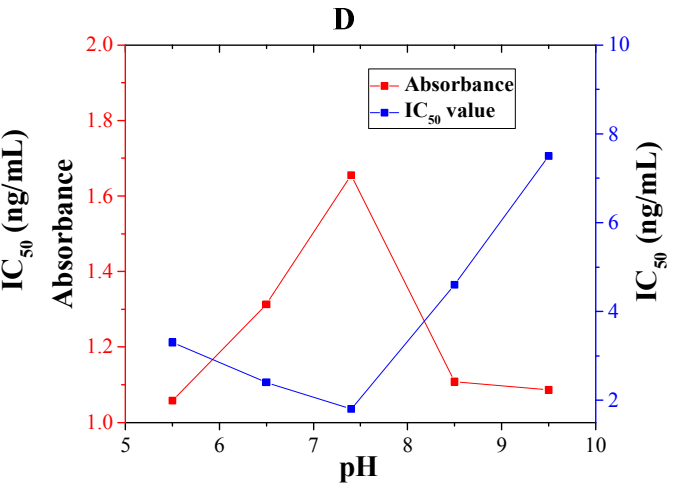

Figure S2. The effects of organic solvents (A) methanol and (B) acetonitrile, (C) ionic strength, and (D) $\mathrm{pH}$ on BA-ELISA performance. 


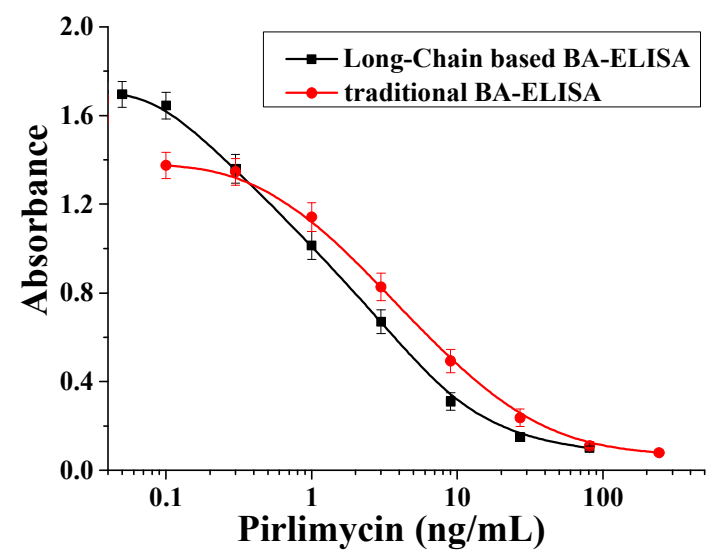

Figure S3. Calibration curves for the long-chain biotin based BA-ELISA and the traditional BAELISA for pirlimycin (1) residue analysis. The data are average values of triplicate samples (average $\pm \mathrm{SD}$ ). The $\mathrm{IC}_{50}$ values were $2.4 \mathrm{ng} / \mathrm{mL}$ and $1.6 \mathrm{ng} / \mathrm{mL}$ for the long-chain biotin based BA-ELISA and the traditional BA-ELISA, respectively. 
A

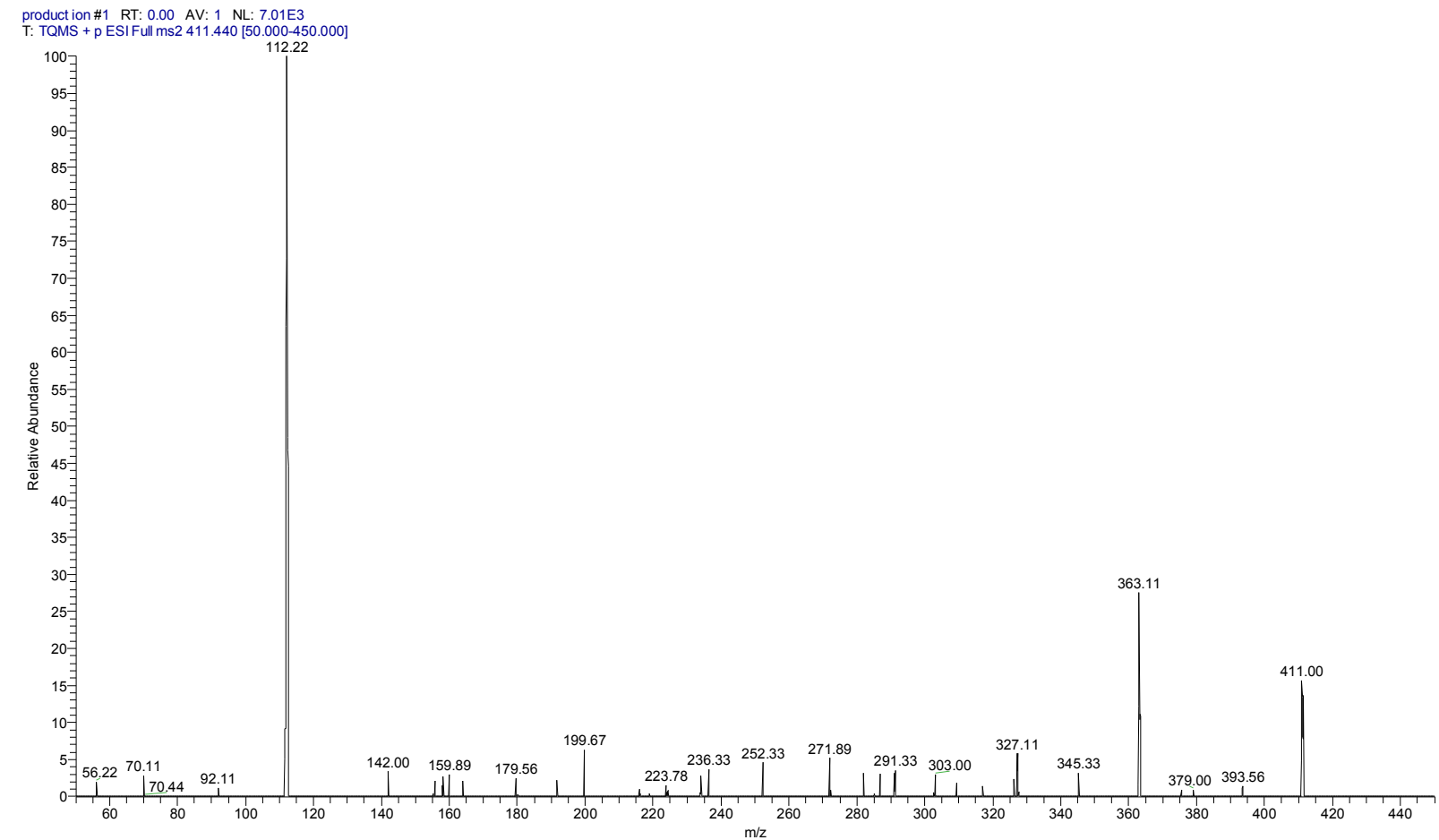

B

std100ppb \#186 RT: 3.09 AV: 1 NL: 5.96E4

T: + CESISPM R2 $411.245[112219-112221,363.144-363.146]$

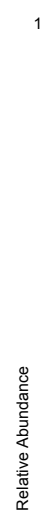

$$
\begin{aligned}
& { }^{100} \exists
\end{aligned}
$$

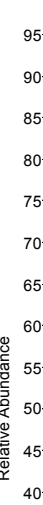

$$
\begin{array}{r}
35 \\
30 \\
25
\end{array}
$$

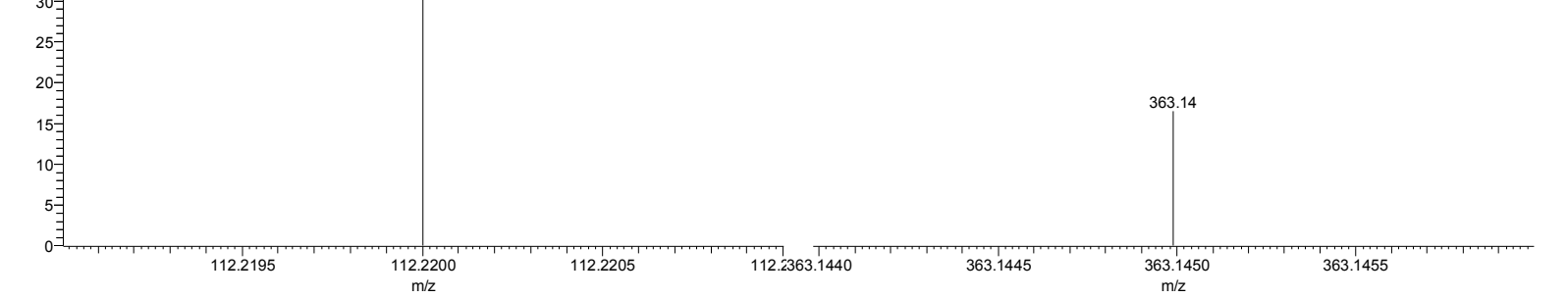

Figure S4. A (A) full scan mass spectrum and a (B) MS/MS spectrum of 1. 

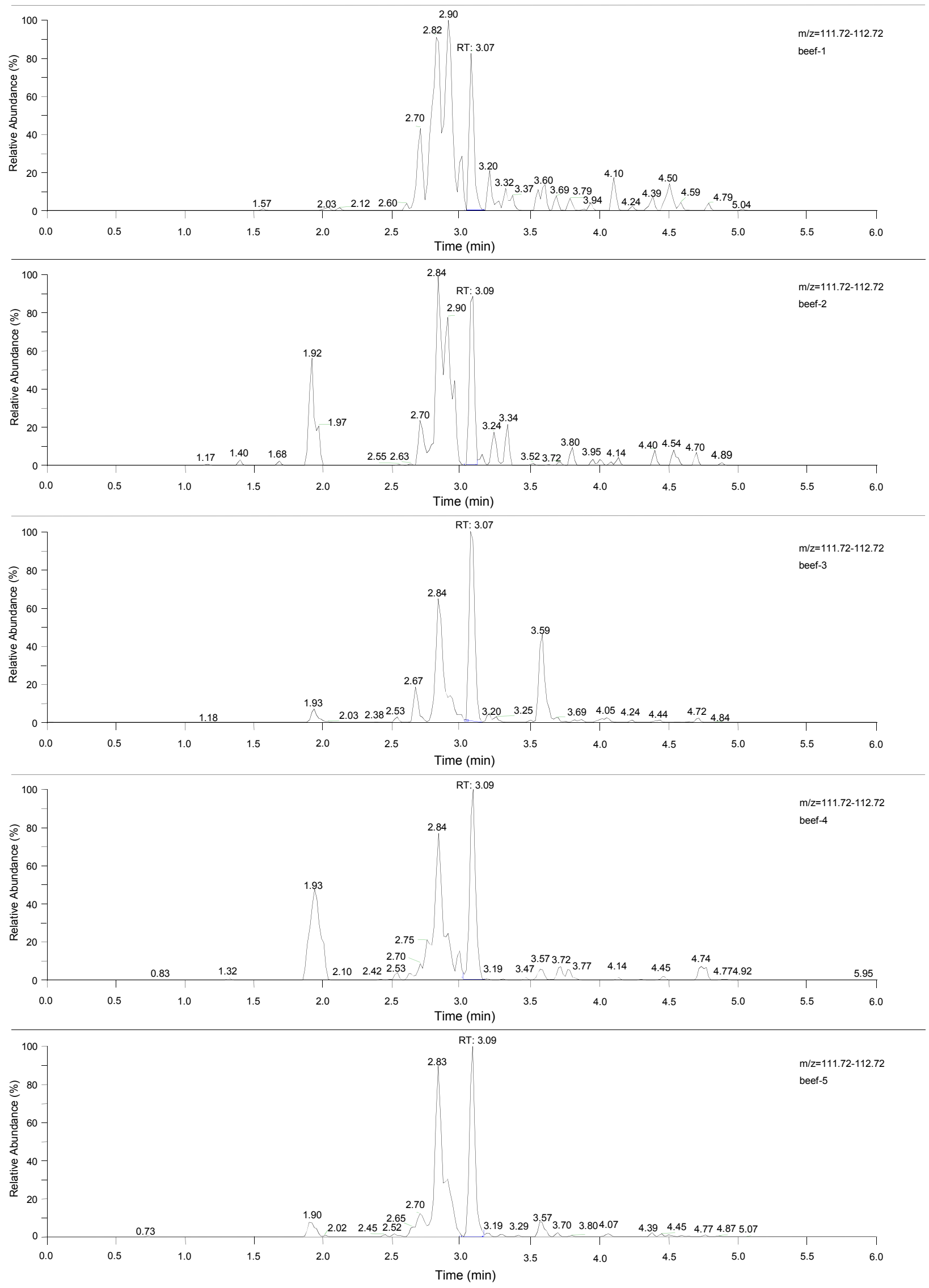

Figure S5. LC-MS/MS analysis of 1 from beef muscle. 

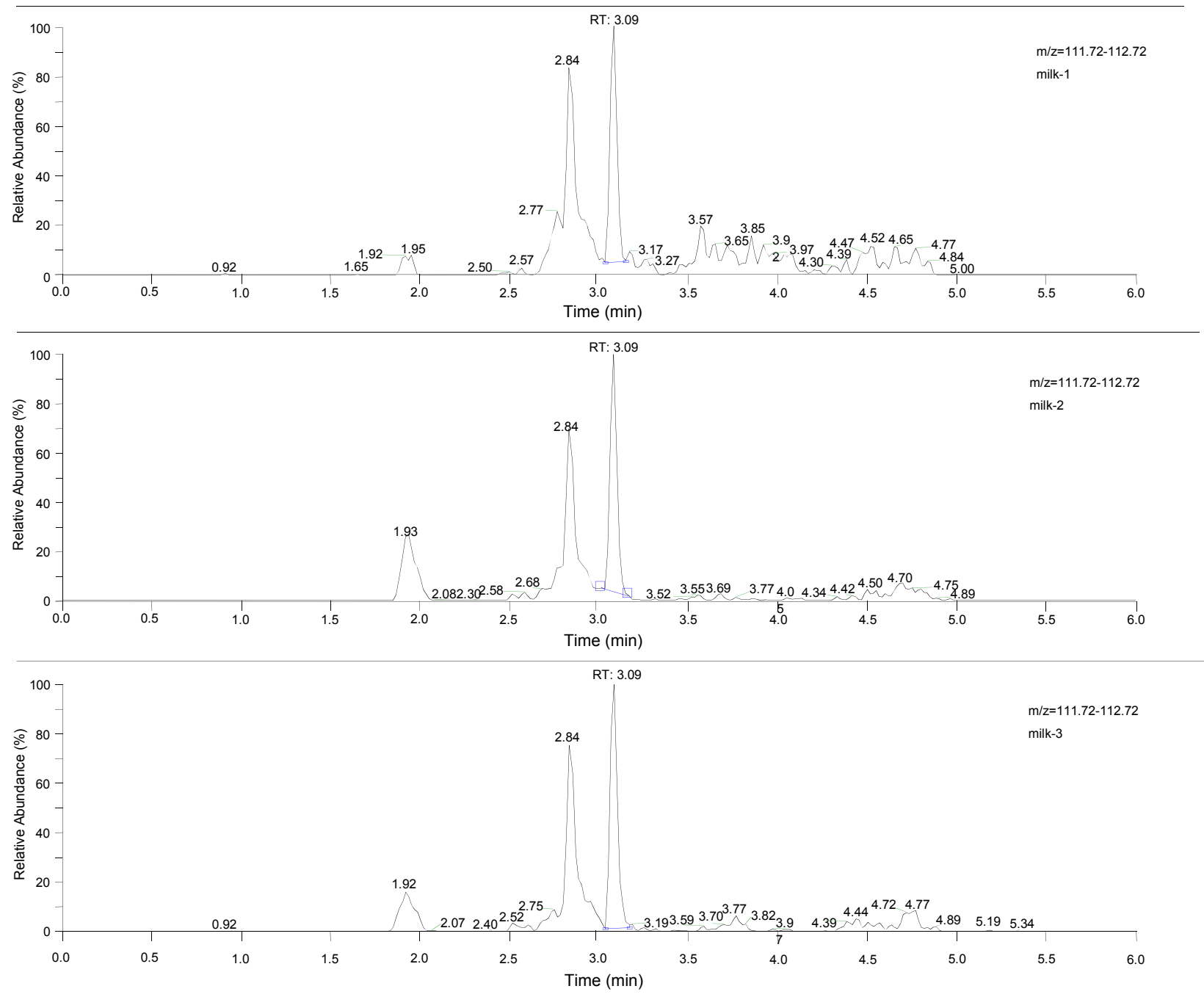

Figure S6. LC-MS/MS analysis of 1 from milk. 

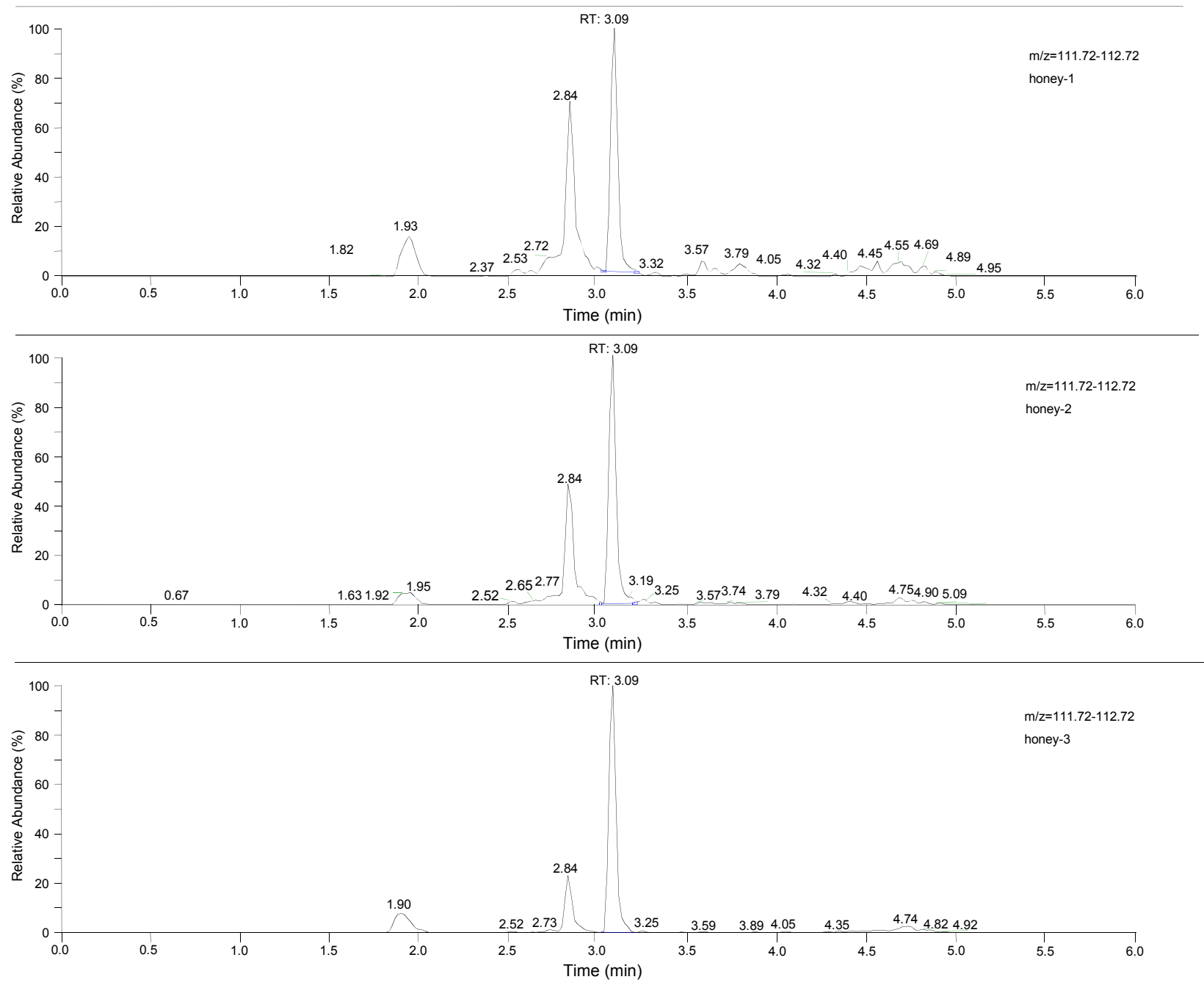

Figure S7. LC-MS/MS analysis of 1 from honey. 
Table S1. Assay Parameters for the Three MAbs Obtained from the Selected Hybridomas.

\begin{tabular}{llccc}
\hline Antibody & & $4 \mathrm{E} 7$ & $6 \mathrm{C} 10$ & $8 \mathrm{H} 12$ \\
\hline Isotype & Subclass & IgG1 & IgG2a & IgG1 \\
& Light chains & $\lambda$ & $\kappa$ & $\lambda$ \\
Affinity (L/moL) & $9.5 \times 10^{7}$ & $2.5 \times 10^{7}$ & $4.5 \times 10^{6}$ \\
$\mathrm{IC}_{50}(\mathrm{ng} / \mathrm{mL})$ & 10.8 & 19.5 & 35.8 \\
\hline
\end{tabular}


Table S2. SRM Parameters of the Target Compound.

\begin{tabular}{llll}
\hline Compound & Precursor $(\mathrm{m} / \mathrm{z})$ & Product $(\mathrm{m} / \mathrm{z})$ & Collision Energy $(\mathrm{V})$ \\
\hline pirlimycin & 411.245 & 112.22 & 30.6 \\
& 411.245 & 363.145 & 18.6 \\
\hline
\end{tabular}

